\title{
Biomineralization of marine-patterned C-scaffolds
}

\section{Cosme Rodríguez-Valencia*}

PhD student, New Materials Group, Applied Physics Department, School of Industrial Engineering, Campus Lagoas-Marcosende, Institute of Biomedical Research of Vigo, University of Vigo, Vigo, Spain

\section{Miriam López-Álvarez}

Doctor, New Materials Group, Applied Physics Department, School of Industrial Engineering, Campus Lagoas-Marcosende, Institute of Biomedical Research of Vigo, University of Vigo, Vigo, Spain

\section{Stefan Stefanov}

PhD student, New Materials Group, Applied Physics Department, School of Industrial Engineering, Campus Lagoas-Marcosende, Institute of Biomedical Research of Vigo, University of Vigo, Vigo, Spain

\begin{abstract}
Stefano Chiussi
Doctor, New Materials Group, Applied Physics Department, School of Industrial Engineering, Campus Lagoas-Marcosende, Institute of Biomedical Research of Vigo, University of Vigo, Vigo, Spain
\end{abstract}

\section{Julia Serra}

Doctor, New Materials Group, Applied Physics Department, School of Industrial Engineering, Campus Lagoas-Marcosende, Institute of Biomedical Research of Vigo, University of Vigo, Vigo, Spain

\section{Pío González}

Professor, New Materials Group, Applied Physics Department, Schoo of Industrial Engineering, Campus Lagoas-Marcosende, Institute of Biomedical Research of Vigo, University of Vigo, Vigo, Spain

Patterned surfaces of marine-derived carbon scaffolds were subjected to a biomimetic approach to be covered with a calcium phosphate thin film. The process was based on Dulbecco's phosphate-buffered saline solution and investigated in different periods of immersion (from hours to days). A complete physicochemical characterization was performed to demonstrate the optimal calcium/phosphorus ratio, thickness and adherence to the substrate of these biomimetic calcium phosphate coatings, which still retained the naturally derived patterning. A chemical mechanism to explain the coating formation has been proposed and documented, based mainly on the presence of carboxylic groups on the C-scaffold surface, what promoted the anchorage of calcium ions at the first stage and the later binding of phosphate groups to calcium ions. The biological response of MC3T3-E1 preosteoblasts on the calcium phosphate-coated scaffolds was investigated to demonstrate the non-cytotoxicity, adequate morphology and spreading of cells after $7 \mathrm{~d}$ of culture, being this proliferation aligned, promoted by the patterning of the scaffold.

\section{Introduction}

Nowadays, the design of 3D scaffolds for the replacement, repair and regeneration of the different human tissues is one of the main pillars and challenges of regenerative medicine. In the case of bone tissue, it is important that its tissue engineering scaffolds present a determined surface chemistry, hierarchical porosity and surface topography that will promote cell attachment and differentiation.

One of the most successful strategies for improving the biocompatibility of the device is to provide it with a calcium phosphate coating resembling, thus, the mineral part of the human bone tissue (hydroxyapatite), which promotes an osteogenic, osteoconductive or osteoinductive activity. However, these bioactive ceramic coatings have also been cited as an efficient strategy to overcome the fibrous tissue encapsulation, a common problem for synthetic metallic and polymeric implants in vivo. ${ }^{1}$

To produce bioceramic coatings, different techniques such as plasma spray, pulsed laser deposition or electron beam evaporation have been intensively studied, but, trying to follow the main postulates that regenerative medicine promotes, the deposition of this calcium phosphate by means of a biomimetic process seems to be the main challenge. Thus, the biomimetic method was first developed by Kokubo $^{2}$ with the purpose of obtaining a calcium phosphate layer by using a simple process based on the immersion of the material (metal, polymer or ceramic) in a simulated body fluid (SBF) with ion concentrations similar to human blood plasma obtaining a bonelike apatite layer on various types of organic polymer and titanium substrates. However, traditional SBF solutions require of lengthy incubation times, typically more than $7 \mathrm{~d}$, to get obtained a uniform calcium phosphate coating. Recent efforts to shorten the time needed for coating have focused on increasing ionic concentrations. Although still a matter of some controversy, the ability of implants to form a calcium phosphate in SBF is object of much interest, and it could give important information on in vivo behavior. ${ }^{3,4}$ The use of Dulbecco's phosphate-buffered saline solutions (DPBS; Sigma-Aldrich, Schnelldorf, Germany) instead of SBF has also been investigated as a biomimetic fluid. Results have been obtained 
with DPBS but with a different perspective since the titanium discs immersed in the DPBS already presented a calcium phosphate coating on them, for example, by electron beam evaporation. ${ }^{5}$ The idea in that case was to incorporate the calcium phosphate coating on other biological or ionic compounds added to the DPBS to increase functionality. The direct immersion of different materials in DPBS to obtain the calcium phosphate layer will be studied in this study.

On the other hand, the biodiversity that characterizes the marine environment represents an enormous potential for obtaining suitable $3 \mathrm{D}$ porous biostructures already patterned in nature. ${ }^{6-8}$ In recent years, several authors have used different marine species (coral skeletons, sea urchins and sponges) as 3D micro- and nano-scaled porous biomatrices where the results confirmed that the 3D topography, and the surface parameters of these materials helped to promote the cell differentiation. ${ }^{9-11}$ The sea rush Juncus maritimus Linnaeus has a vascular system uniformly distributed throughout its section that provides a hierarchical interconnected macro-, micro- and nanoporosity distributed along the entire plant. As an added value, this plant presents a double surface patterning in the upper epidermal layers aligned in the direction of the plant's growth. The supporting material proposed in this study to be immersed in DPBS will consist on a bioinspired carbon scaffold (C-scaffold) obtained from J. maritimus, with hierarchical porosity and surface topography maintained from the original plant that has already been proved to promote cell attachment and alignment as well as an important osteogenic activity. ${ }^{10-12}$

The intrinsic properties of these marine C-scaffolds together with the strong biocompatibility of calcium phosphate coatings have inspired the design of the bioceramic presented in this study. Thus, the aim of this study was to obtain a calcium phosphate layer on the porous C-scaffold obtained from J. maritimus by a simple biomimetic method using DPBS solution. The physicochemical study, the fundaments of the calcium phosphate anchorage on the surface scaffold and a preliminary biocompatibility evaluation of the calcium phosphatecoated scaffold by using preosteoblastic cells are presented.

\section{Materials and methods}

\subsection{Preparation of C-scaffolds}

The cylindrical scaffolds were obtained as follows: The sea rush J. maritimus was subjected to air drying for several days and then introduced in a pyrolysis furnace where the thermal decomposition was carried out by a gradual increase in temperature of $2^{\circ} \mathrm{C} / \mathrm{min}$ up to $500^{\circ} \mathrm{C}$. The furnace was then maintained at that temperature for $10 \mathrm{~min}$, followed by a gradual decrease of $20^{\circ} \mathrm{C} / \mathrm{min}$ down to room temperature. The obtained carbon samples were cut to obtain pieces of $15 \mathrm{~mm}$ in length and $2 \mathrm{~mm}$ in diameter. Finally, to remove the remaining marine salts (sodium chloride and potassium chloride) scaffolds were subjected to ultrasonic baths of $60 \mathrm{~min}$ with warm milli-Q water, renewing it every $15 \mathrm{~min}$ and dried at room temperature. More details on the scaffold fabrication can be found in previously published works. ${ }^{10,14}$

\subsection{Biomimetic process}

Containers used for holding and immersing the samples were ultrasonically washed with milli-Q water followed by $\mathrm{HCl} 1 \mathrm{~N}$ and, again, milli-Q water to, finally, let them dry at room temperature. On the other hand, the carbon samples were cleaned in ultrasound baths with milli-Q water during $60 \mathrm{~min}$. Then, C-scaffolds were placed in the upright position in the containers and immersed with $50 \mathrm{ml}$ of DPBS (Sigma Aldrich) at $60 \cdot 0 \pm 0 \cdot 5^{\circ} \mathrm{C}$ for $6 \mathrm{~h}$ and $7 \mathrm{~d}$, respectively. After each incubation time, samples were rinsed with warm milli-Q water during $40 \mathrm{~min}$ and air dried slowly at room temperature.

\subsection{Physicochemical characterization}

The morphology and semiquantitative elemental composition of the C-scaffold, before and after the biomimetic process were studied under a scanning electron microscope (SEM) Philips XL30 (Eindhoven, The Netherlands) equipped with an energy-dispersive X-ray spectroscope (EDS; Eindhoven, The Netherlands). EDS was used to confirm the presence of calcium and phosphorous ions and to elicit information regarding the calcium/phosphorus ratio of the biomimetic coatings. The pore distribution was measured by mercury porosimetry with an Autopore IV 9500 Micromeritics, Norcross, USA (University of Santiago). The thickness of the calcium phosphate biomimetic coating was evaluated in transversal and longitudinal section by introducing them in resin, followed by polishing of the cylindrical sample and the evaluation of the interface between the C-scaffold and the calcium phosphate coating by using back scattering electron detector (BSE; Eindhoven, The Netherlands) and the software Image J (Java, National Institutes of Health, USA). Six measures were taken in different points of one section, at different sections and samples. The Fourier transform infrared (FTIR) spectra were collected using a Thermo Nicolet 6700 Fourier transformed infrared spectrometer (Madison, USA) in the range $4000-450 \mathrm{~cm}^{-1}$. The FTIR spectra were obtained in transmission mode by using pressed potassium bromide tablets. These tablets were prepared by grinding the sample $(2 \mathrm{mg})$ with a completely dehydrated potassium bromide $(200 \mathrm{mg})$ in an agate mortar (5-10 min). To complement the study, effusion measurements with a heating ramp of $2^{\circ} \mathrm{C} / \mathrm{min}$ were carried out on the C-scaffold.

\subsection{In vitro cell culture}

A cell suspension of the preosteoblastic line MC3T3-E1 (ECACC, UK) of $1.7 \times 10^{5}$ cells $/ \mathrm{ml}$ in $100 \mu \mathrm{l}$ of MEM-alpha (SigmaAldrich, St. Louis, USA) supplemented with $10 \%$ fetal bovine serum (FBS; Invitrogen, USA) was added directly on the surface of the calcium phosphate-coated scaffolds, which were placed in 96-well tissue culture plates. Cells were cultured for $7 \mathrm{~d}$ in a humidified atmosphere with $5 \%$ carbon dioxide and at $37^{\circ} \mathrm{C}$. The culture medium was renewed every $2-3 \mathrm{~d}$. 
Bioinspired, Biomimetic and Nanobiomaterials Volume 3 Issue BBN2
Biomineralization of marine-patterned

C-scaffolds

Rodríguez-Valencia et al.
Cell morphology was analyzed by SEM. Three replicates were fixed and dehydrated, as described elsewhere, ${ }^{13}$ to be analyzed using Philips XL30 and FEG JEOL JSM 6700F (Tokyo, Japan). The cellular cytoskeleton was observed by confocal laser scanning microscopy (CLSM). After $7 \mathrm{~d}$ of incubation time, cells were fixed with a solution of paraformaldehyde as described elsewhere. ${ }^{10}$ Alexa fluor 488 phalloidin (Life Technologies, California, USA) and propidium iodide (Sigma-Aldrich, St. Louis, USA) solutions were added to visualize the cellular cytoskeletal actin filaments and cell nuclei, respectively. Cells were observed with a confocal microscope Bio-Rad MRC 1024 (California, USA).

\subsection{Statistical analysis}

The data of the thicknesses of the calcium phosphate layers were obtained through scanning electron micrographs in six random fields and were averaged for each sample. Data are indicated as mean \pm standard deviation. The significant differences were assessed with the probability associated with two-tailed Student's $t$-test.

\section{Results and discussion}

\subsection{C-scaffolds" microstructure}

Figure 1(a)-1(c) shows the microstructure of the marine plant J. Maritimus by SEM micrographs, where a highly porous structure can be observed distributed through the whole section of the plant even to the outer layers in an interconnected network. Figure 1(b) presents higher-magnified images of the pores of 20-25 $\mu \mathrm{m}$, that correspond to the xylem, and of around $5 \mu \mathrm{m}$, the phloem, more clearly shown in Figure 1(c). Figure 1(d)-1(f) shows the microstructure of the bioinspired C-scaffold obtained from this marine plant where the preservation of the original porosity of the sea rush can be observed with hierarchical distribution of pore sizes disposed through the whole section of the carbon (Figure 1(d)), with pores of 20-25 $\mu \mathrm{m}$ (Figure 1(e)) and around $5 \mu \mathrm{m}$ (Figure 1(e) and more clearly in Figure 1(f)) perfectly preserved.

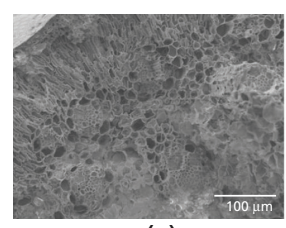

(a)

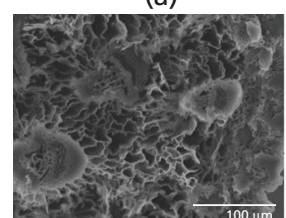

(d)

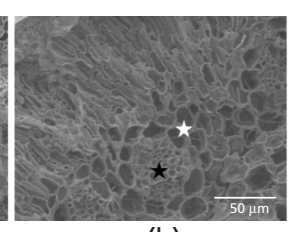

(b)

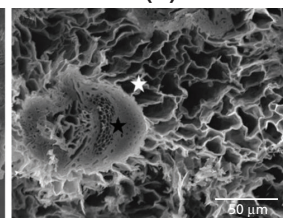

(e)

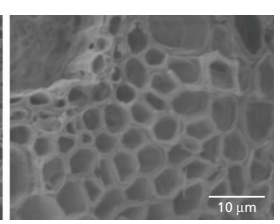

(c)

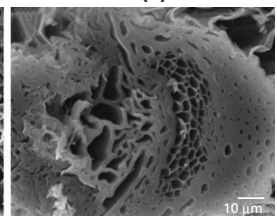

(f)
Figure 1. Scanning electron micrographs in cross-section at different magnifications of natural Juncus maritimus $(\mathrm{a}-\mathrm{c})$ and carbon scaffolds $(d-f)$. White star indicates xylem and black star phloem
Figure 2, previously reported in Ref. 10, presents the pores' distribution of these bioinspired C-scaffolds obtained by mercury porosimetry. A three-modal distribution is shown with macropores (80-150 $\mu \mathrm{m}$ in diameter), mesopores $(1-10 \mu \mathrm{m})$ and nanopores $(0 \cdot 03-0 \cdot 2 \mu \mathrm{m})$ with higher volume of pores of 3 and $8 \mu \mathrm{m}$ followed by $0.15 \mu \mathrm{m}$ and of around $140 \mu \mathrm{m}$. The per cent of total porosity resulted in a value of $63 \%$. These results of porosity give this material a great potential as scaffold since that high porosity levels will support migration and proliferation of osteoblasts and mesenchymal cells, bone tissue ingrowth, vascular invasion, nutrient delivery and matrix deposition in empty spaces. In fact, the presence of macroporosity (size $>100 \mu \mathrm{m}$ ) has a critical impact on osteogenic outcomes, promotion of vascularization and mass transportation of nutrients and waste products. ${ }^{15}$ Meso- and microporosity, size around $10 \mu \mathrm{m}$, favor capillary formation and, finally, nanoporosity allows diffusion of molecules for nutrition and signalling $^{16}$, and it has been demonstrated to favor the adsorption of proteins for the anchorage of the cells. ${ }^{17,18}$

Figure 3 presents the surface patterning of the marine plant and the $\mathrm{C}$-scaffold by SEM micrographs. The macropatterning of the natural plant with ridges of around $100 \mu \mathrm{m}$ in width oriented in the plant's growth direction (Figure 3(a)) containing a micropatterning with ridges of around $7 \mu \mathrm{m}$ oriented in the same direction (Figure 3(b)) are shown. Arranged in rows, the stomata can be observed (in Figure $3(a)$ ), which corresponds to oval-shaped pores used by the plant to regulate the gas exchange and water loss. The double macro- and micro-scaled patterning and the stomata appeared perfectly preserved or even enhanced on the C-scaffolds (Figure 3(c) and 3(d)). This surface patterning makes of this scaffold as a very promising structure to promote cell alignment (as previously referred in Ref. ${ }^{10}$ ). At the same time, it has been demonstrated that the surface topography, at the micrometer and submicrometer level, affects the attachment, proliferation and differentiation of cells on biomaterials. ${ }^{19-21}$ An increase in surface porosity and roughness of biomaterials improves cellular attachment, proliferation, differentiation and bone-ingrowth ${ }^{17-22}$; thus,

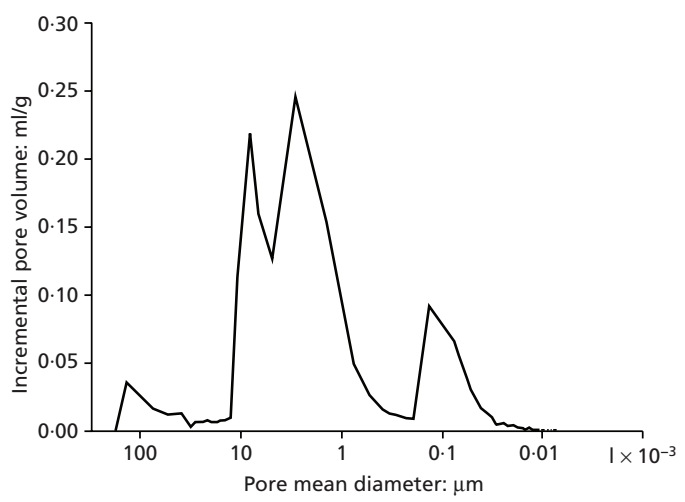

Figure 2. Pore size distribution obtained by mercury porosimetry of carbon scaffolds (data modified from Ref. ${ }^{10}$; http://dx.doi. org/10.1088/1748-6041/6/4/045012; reproduced by permission of IOP Publishing. All rights reserved) 
Bioinspired, Biomimetic and Nanobiomaterials Volume 3 Issue BBN2
Biomineralization of marine-patterned

C-scaffolds

Rodríguez-Valencia et al.

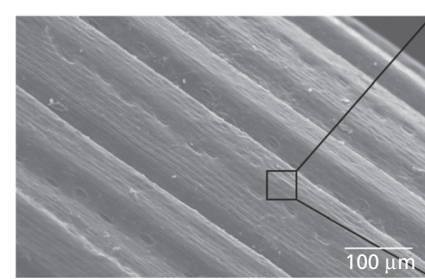

(a)

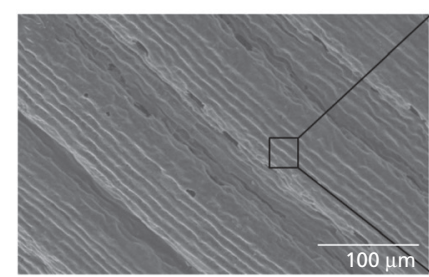

(c)

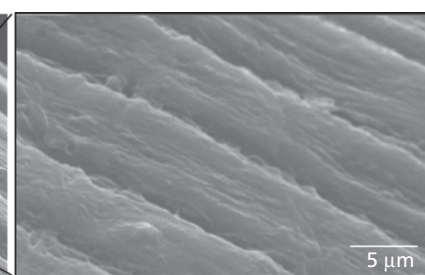

(b)

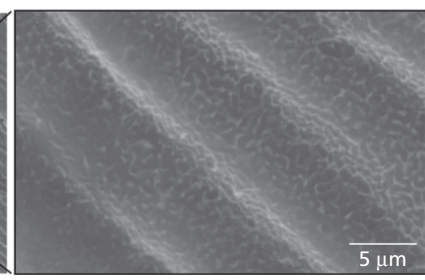

(d)
Figure 3. Scanning electron micrographs at different magnifications of the surface for natural Juncus maritimus (a-b) and its corresponding carbon scaffold (c-d)

the presence of the stomata in the scaffold can help the new formed tissue to better attach to the scaffold by this bone-ingrowth.

In order to deepen on the characterization of this C-scaffold, FTIR and effusion measurements were carried out. Figure 4 presents the FTIR spectrum of the scaffold where the main vibration modes were identified as follows:

- Hydrocarbons groups located between 3044 and $2760 \mathrm{~cm}^{-1}$. The C-H groups exhibited sharp peaks located between 2800 and $2960 \mathrm{~cm}^{-1}$ associated to $\mathrm{CH}_{2}$ symmetric stretching (2860-2875 $\left.\mathrm{cm}^{-1}\right), \mathrm{CH}_{2}$ asymmetric stretching (2910-2930 $\mathrm{cm}^{-1}$ ) and $\mathrm{C}-\mathrm{H}_{3}$ asymmetric stretching $\left(2950-2960 \mathrm{~cm}^{-1}\right) .{ }^{23-}$ ${ }^{25}$ Furthermore, the peak at $873 \mathrm{~cm}^{-1}$ attributed to C-H out of plane bending in benzene derivatives. ${ }^{26} \mathrm{In}$ fact, well-resolved peak emerged at 1380 attributed to $\mathrm{C}-\mathrm{H}_{3}$ methyl umbrella bending mode. ${ }^{23}$

- Oxygen-containing functional groups ${ }^{27}$ such as $\mathrm{C}=\mathrm{O}(1700$ $\left.1765 \mathrm{~cm}^{-1}\right)$ and C-O $\left(1230-1100 \mathrm{~cm}^{-1}\right)$. The absorption band of $\mathrm{OH}$ bonds, found in the range of $3600-3400 \mathrm{~cm}^{-1}$, is due to the adsorbed molecules of water. ${ }^{28}$ At the same time, the absorption bands found at 2388 and $2278 \mathrm{~cm}^{-1}$ are attributed to the carbon dioxide adsorbed from the atmosphere. ${ }^{29} \mathrm{On}$ the other hand, the broad $\mathrm{OH}$ stretch band between 3080 and $2980 \mathrm{~cm}^{-1}$ could correspond to carboxylic acid present at the surface of the $\mathrm{C}$-scaffold ${ }^{23}$ as occurs with the weaker band at $1735 \mathrm{~cm}^{-1}$ associated to a $\mathrm{C}=\mathrm{O}$ stretching mode and with the band at $1110 \mathrm{~cm}^{-1}$ attributed to $\mathrm{C}-\mathrm{O}$ stretching mode. The band at $1430 \mathrm{~cm}^{-1}$ position could be attributed to $\mathrm{OH}$ in plane bending and the peak at $750 \mathrm{~cm}^{-1}$ is associated to carbon monoxide bending vibration in-plane. ${ }^{23,29}$ Furthermore, the peaks in the region of 1230 and $1210 \mathrm{~cm}^{-1}$ indicate the appearance of aromatic phenyl C-O. ${ }^{30}$

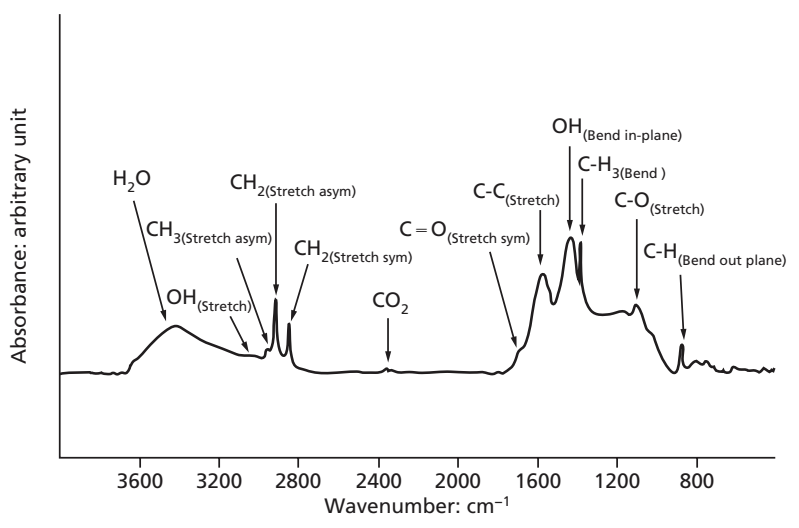

Figure 4. Fourier transform infrared spectrum of carbon scaffold before being immersed in Dulbecco's phosphate-buffered saline solution

Concerning the effusion experiment (Figure 5), the decomposition temperature of the C-scaffold and the partial pressures obtained, allowed us to assess the nature of the groups on its surface. The main functional groups were thermally decomposed at different temperatures during the effusion experiment releasing first water vapor around $100^{\circ} \mathrm{C}$, carbon monoxide and carbon dioxide at $400^{\circ} \mathrm{C}$, again carbon monoxide, water vapor and little of carbon dioxide at around $550^{\circ} \mathrm{C}$ and, finally, around hydrogen at $650^{\circ} \mathrm{C}$. Regarding the thermal decomposition studies, it has been already published ${ }^{30}$ that the most of the oxygen complexes on the carbon's surface, decompose as carbon dioxide and carbon monoxide. At the same time, the amounts and temperatures at which these gases appeared are characteristic of the oxygen-containing surface groups, and it is proven that the carbon dioxide and carbon monoxide desorption profiles correspond to the decomposition of the carboxylic anhydride group.

\subsection{Biomimetic calcium phosphate coating}

Figure 6 shows the surface of the $\mathrm{C}$-scaffolds after being immersed in DPBS solution at $60^{\circ} \mathrm{C}$ for $6 \mathrm{~h}$ (Figure 6(a)-6(c)) and $7 \mathrm{~d}$ (Figure 6(d)-6(f)) by SEM micrographs at three different magnifications. It can be observed as after $6 \mathrm{~h}$ of incubation a homogeneous calcium phosphate layer appeared covering the complete surface perfectly adapted to the patterning surface of these C-scaffolds (Figure 6(a)). Even the inside of a stoma seems to be filled (Figure 6(b)). The calcium phosphate layer was composed by random clusters of nanoflakes where the needle-like particles could be perfectly appreciated (Figure 6(c)). After 7 d, (Figure 6(d)-6(f)), the layer was again covering the entire surface of the scaffold, adapting to the macro- and micro-patterning and to the surface topography, preserving it. The stoma was completely filled and covered by the layer of calcium phosphate (Figure 6(e)). The incubation time did not affect the morphology of the calcium phosphate layer formed on the surface of the $\mathrm{C}$-scaffolds, which presented, in both cases (6 h and $7 \mathrm{~d}$ ), a morphology based on nanoclusters randomly organized (Figure 6(c) and 6(f)). 
Bioinspired, Biomimetic and Nanobiomaterials Volume 3 Issue BBN2
Biomineralization of marine-patterned

C-scaffolds

Rodríguez-Valencia et al.
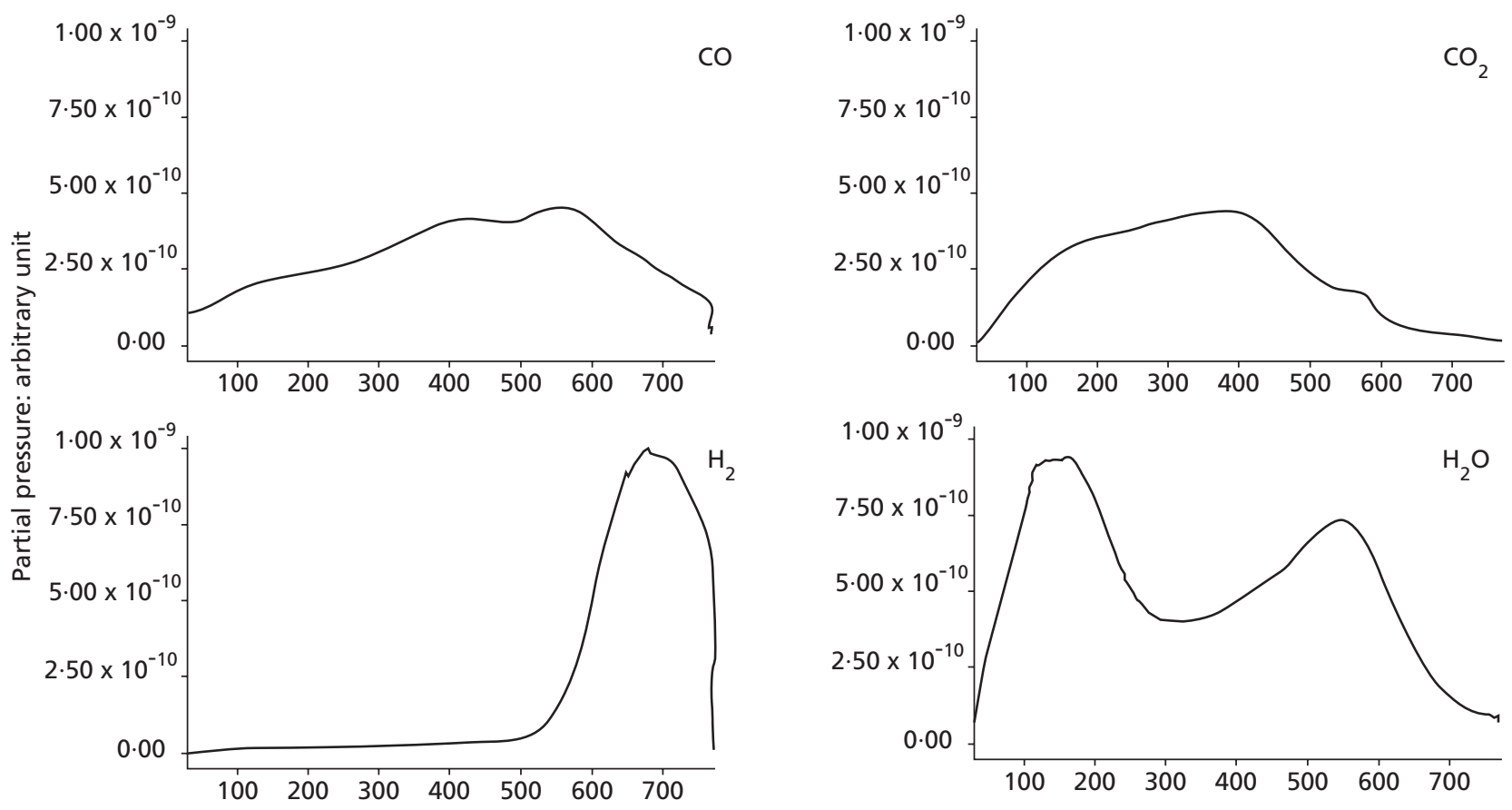

Temperature: ${ }^{\circ} \mathrm{C}$

Figure 5. Partial pressures of carbon monoxide, carbon dioxide,

hydrogen and water from effusion measurements of carbon scaffold

before being immersed in Dulbecco's phosphate-buffered saline solution

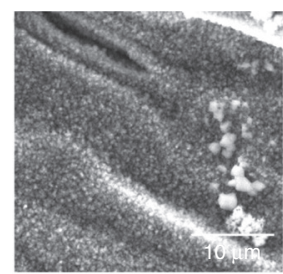

(a)

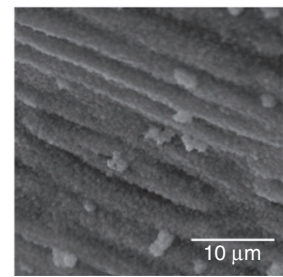

(d)

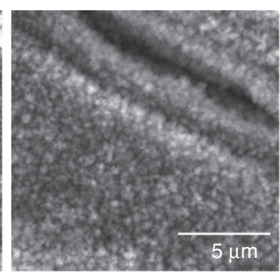

(b)

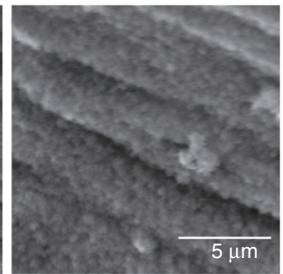

(e)

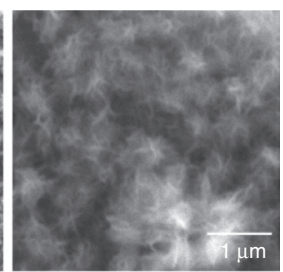

(c)

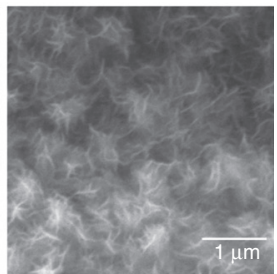

(f)

Figure 6. Scanning electron micrographs of the surface of the carbon scaffolds immersed in Dulbecco's phosphate-buffered saline solution solution for $6 \mathrm{~h}(\mathrm{a}-\mathrm{c})$ and $7 \mathrm{~d}(\mathrm{~d}-\mathrm{f})$ at three magnifications

Figure 7 presents the C-scaffold immersed in DPBS for $7 \mathrm{~d}$ in a detail of a longitudinal section by SEM micrographs (Figure 7(a) and 7(b)) and the EDS spectra of C-scaffolds after immersion in DPBS solution for $6 \mathrm{~h}$ (Figure 7(c) and 7(d)). Figure 7(a) shows the calcium phosphate coating, that was only distributed

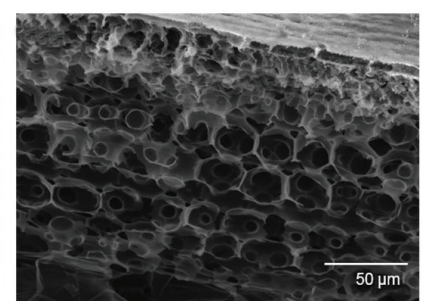

(a)

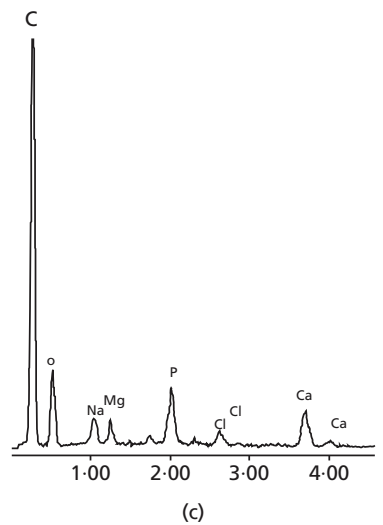

Figure 7. Scanning electron micrographs of calcium phosphate C-scaffolds in longitudinal section after $7 \mathrm{~d}$ of immersion in Dulbecco's phosphate-buffered saline solution solution $(a, b)$ and energydispersive X-ray spectroscope spectra after $6 \mathrm{~h} \mathrm{(c)}$ and after $7 \mathrm{~d}(\mathrm{~d})$. 
at the surface of the scaffold, maintaining intact the impressive interconnected and hierarchical porosity of the inside of the C-scaffold. In Figure 7(b), detail of the calcium phosphate coating is presented where an entire and compact layer can be clearly appreciated. To confirm the presence of calcium and phosphorous ions and to elicit information regarding the calcium/phosphorous ratio of the biomimetic coatings, EDS analysis was performed (Figure 7(c) and 7(d)). Figure 7(c) presents the spectra obtained for the surface of C-scaffold immersed in DPBS for $6 \mathrm{~h}$ and Figure 7(d) for $7 \mathrm{~d}$. As it can be observed, both EDS spectra revealed the presence of calcium and phosphate elements on both coatings. The carbon signal came from the scaffold itself. Furthermore, EDS semiquantitative measurements established with the ratios between calcium and phosphorous intensities detected, showed approximate values for calcium/phosphorus ratio of 0.63 for $6 \mathrm{~h}$ coating and of 0.93 for $7 \mathrm{~d}$.

The most searched calcium/phosphorus ratio found in literature for many years has been $1 \cdot 67$, what corresponds to stoichiometric hydroxyapatite. This material has been widely used as bone graft or as coatings on metal alloys to improve bone growth around orthopedic implants. The ratio of 1.5 corresponds to tricalcium phosphate, extensively used as drug delivery agents or injectable cements for filling bone defects. It has been recently published ${ }^{31}$ that an in vitro study of the calcium/phosphorus stoichiometry influence in different applications of calcium phosphate ceramics such as coatings or as bone cements for filling defects. Thus, different calcium/phosphorus ratios $(0.5,1.0,1.5,2.5)$ were tested, and the more appropriate response as a coating was found for calcium/phosphorus ratio 1.0 with higher viability of osteblasts together with significant highest alkaline phosphatase activity values. Thus, the ratio of 0.93 obtained for the calcium phosphate layer on the C-scaffold at the present work after immersion in DPBS for $7 \mathrm{~d}$ corresponds perfectly with the intended application as a coating.

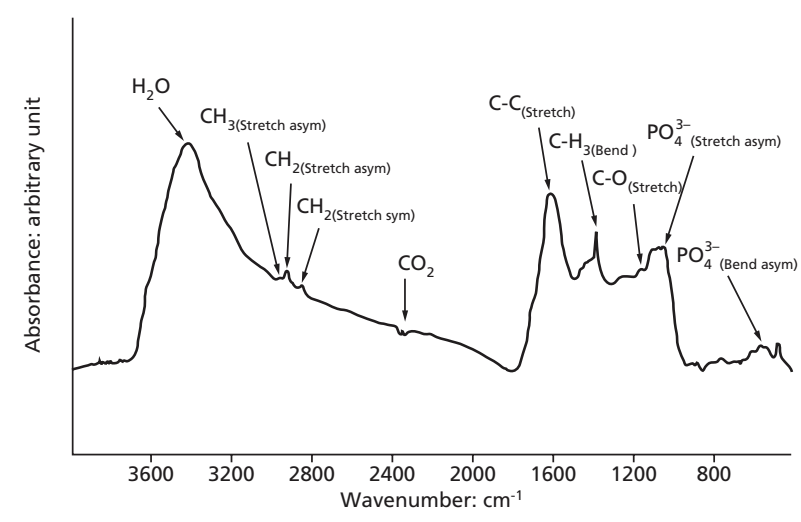

Figure 8. Fourier transform infrared spectrum of carbon scaffold after being immersed in Dulbecco's phosphate-buffered saline solution
At the same time, the thicknesses of the calcium phosphate coatings were estimated, as previously mentioned, by analyzing the resinimmersed coated C-scaffolds in cross-section with BSE, and then measurements were performed with the Image J software. Thus, the calcium phosphate layers thicknesses were of $0 \cdot 8 \pm 0 \cdot 2 \mu \mathrm{m}$ and $0 \cdot 9 \pm 0 \cdot 2 \mu \mathrm{m}$ after $6 \mathrm{~h}$ and $7 \mathrm{~d}$ of DPBS immersion, respectively, without a statistically significant difference between them. As a result of this, the authors concluded that the growth of the calcium phosphate coating, in a static process, was already stabilized after $6 \mathrm{~h}$ of immersion probably due to the depletion of ions from the DPBS solution.

Figure 8 presents the FTIR measurement carried out on the C-scaffold after immersion in DPBS to characterize the coating on the scaffold. Thus, as it can be observed, it was found that the typical stretching (at $1054 \mathrm{~cm}^{-1}$ ) and bending (at $605 \mathrm{~cm}^{-1}$ ) peaks attributed to phosphate groups. Moreover and in agreement with the results previously reported at Figure 4, the spectrum presented the bands already identified for the uncoated C-scaffold.

Finally, and in order to understand the mechanism of the calcium phosphate anchorage on the surface of the $\mathrm{C}$-scaffold, the results of the FTIR and effusion experiments together with literature have been discussed as follows: Thus, C-scaffolds were obtained after a pyrolysis up to $500^{\circ} \mathrm{C}$ in oxygen atmosphere. This heat treatment in an oxygen atmosphere increased the concentration of the oxygen groups on the surface of carbon samples ${ }^{30}$ and that temperature caused the decomposition of carboxyl groups of the surface. ${ }^{29}$ After pyrolyzation, the C-scaffolds were subjected to a cleaning process in distilled water, which promoted the subsequent hydrolysis and the formation of the carboxylic acid groups (already shown in FTIR spectra at Figure 4) by means of the anhydride carboxylic groups. As it was previously published by Otake et al.,,$^{30}$ those carboxylic acid groups present on the $\mathrm{C}$-surface can be neutralized by divalent ions as, in that case, barium hydroxide. Moreover, and concerning with the formation mechanisms of a calcium phosphate coating through in vitro immersion in SBF proposed by Kokubo et al. ${ }^{32}$, they demonstrated that functional groups with negative charge on the substrate will favor the nucleation and accumulation of ions from the SBF with positive charge promoting, at this way, the first bond to obtain

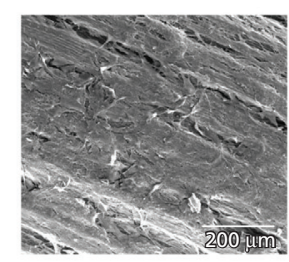

(a)

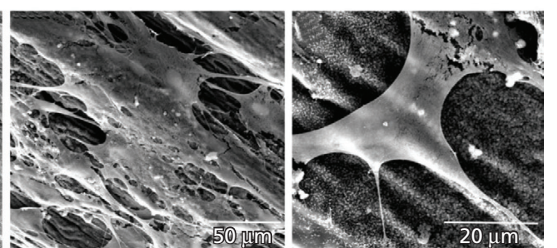

(b)
Figure 9. Scanning electron micrographs at three different magnifications of MC3T3-E1 in vitro test on C-scaffold after $7 \mathrm{~d}$ of phosphate-buffered saline incubation 
Bioinspired, Biomimetic and Nanobiomaterials Volume 3 Issue BBN2
Biomineralization of marine-patterned

C-scaffolds

Rodríguez-Valencia et al.

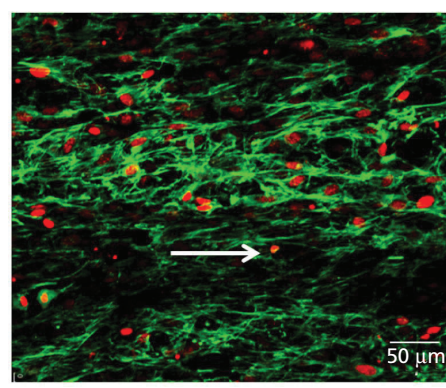

(a)

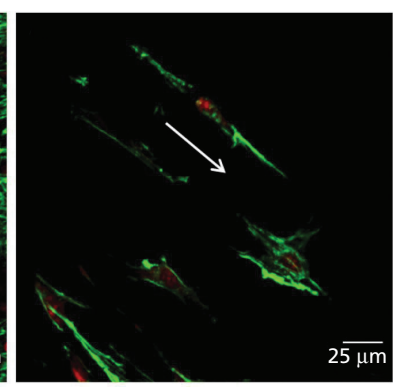

(b)
Figure 10. Morphological evaluation by confocal laser scanning microscopy of MC3T3-E1 cells on C-scaffold after $7 \mathrm{~d}$ of incubation. (a) Maximum projection image and (b) an image obtained going in a certain depth in the $z$ axis. Cell nuclei and F-actin filaments were stained in red and green, respectively

the bone-like apatite layer. In the case of carbon materials, the presence of carboxyl groups make the surface negatively charged, as it has been already proven ${ }^{33}$ on the functionalized carbon nanotubes. Therefore, by combining these theories with FTIR spectroscopy results and effusion experiments, the authors have elucidated that the negatively charged surface of acidic oxygen of the C-scaffolds can be neutralized after immersion in DPBS solution by combining electrostatically with the dissolved $\mathrm{Ca}^{2+}$ ions at the DPBS solution. Later, while the concentration of $\mathrm{Ca}^{2+}$ ions at the $\mathrm{C}$-scaffold surface increases, the neutral charge will become positive starting, then, the electrostatic attraction of negatively charged $\mathrm{PO}_{4}^{3-}$ ions, again from DPBS, to form the calcium phosphate layer bonded to the surface of the $\mathrm{C}$-scaffold.

\subsection{Coatings biocompatibility}

In order to check the biocompatibility of these calcium phosphate-coated C-scaffolds, samples were incubated with the MC3T3-E1 preosteoblastic cell line for $7 \mathrm{~d}$. Figure 9 presents the cells morphology on the surface after $7 \mathrm{~d}$ of incubation in three different magnifications. As it can be observed in Figure 9(a), almost the whole surface has been covered by a layer of cells that have proliferated by extending their filopodia mainly in the direction marked by the patterning of the surface of the C-scaffold (Figure 9(b)) and with the characteristic flat morphology of this osteoblast-like cells adapting perfectly to the surface topography (Figure 9(b) and 9(c)). It is clearly observed in Figure 9(c) that the calcium phosphate coating is still covering the whole surface after $7 \mathrm{~d}$ of incubation and how the cells still aligned in the pattern direction, as occurred in the uncoated scaffold described by López-Álvarez et al. ${ }^{10}$

The cytoskeleton of the cells after $7 \mathrm{~d}$ of incubation was analyzed by CLSM (Figure 10). The cell nuclei are seen in red and the actin filaments of the cytoskeleton in green. In Figure 10(a), the oriented actin fibers, as well as the nuclei, can be clearly observed. The completely covered layer of cells can be appreciated and also, as their actin filaments are preferentially oriented in the direction of the patterning of the material (indicated by the white arrow) and in both the lower parts of the profile (grooves) and the higher ones (ridges). It is an interesting fact that the coating did not modify at all the surface patterning of these scaffolds so that they continue promoting the cell alignment as it did in the uncoated scaffold previously published. ${ }^{10}$ When going in depth in the $z$ axis through the coating and at higher magnification, several images of isolated cells could be observed and again they were growing by following the direction of the patterning of the scaffold (Figure 10(b)).

\section{Conclusions}

The viability to obtain calcium phosphate coatings on C-scaffolds from marine sources using a biomimetic method was demonstrated. A homogeneous calcium phosphate layer, composed by random clusters of nanoflakes with needle-like particles, with around $1 \mu \mathrm{m}$ of thickness was obtained in short periods of time (hours). The calcium/phosphorus ratio obtained after immersion in DPBS for $7 \mathrm{~d}$ was of 0.93 , which is within the values recommended for a better osteoblastic behavior in coating applications. The mechanism of calcium phosphate engagement on the C-scaffolds surface has been concluded as follows: negatively charged surface of $\mathrm{C}$-scaffolds are neutralized by combining electrostatically with $\mathrm{Ca}^{2+}$ ions from DPBS solution and while the concentration of $\mathrm{Ca}^{2+}$ ions at the $\mathrm{C}$-scaffold surface increases, the neutral charge will become positive, initiating then the electrostatic attraction of negatively charged $\mathrm{PO}_{4}^{3-}$ ions, again from DPBS, to form the calcium phosphate coatings. Finally, their biocompatibility was demonstrated by preliminary in vitro studies where preosteoblastic cells (MC3T3-E1) that presented healthy morphology and proliferated by covering the entire scaffold. A good adherence of the calcium phosphate coatings during the $7 \mathrm{~d}$ of cell incubation was observed together with the cell alignment. This study demonstrated the viability to develop and assemble in a single device the excellent osteoconductive properties inherent in the $\mathrm{C}$-scaffold with the osteoinductive properties provided by the surface patterning together with the calcium phosphate coating.

\section{Acknowledgements}

This study was partially financed by POCTEP 0330IBEROMARE1P project, FEDER MARMED Atlantic Area Transnational Programme, Xunta de Galicia (GRC2013-008), Fundación Mutua Madrileña (Project 2013/14) and Ministerio de Ciencia e Innovación (MAT 2010-18281). The whole techniques (except porosimetry equipment and effusion measurements) were provided by the Support Centre for Scientific and Technological Research (CACTI, University of Vigo). M. López-Álvarez and S. Stefanov thank the funding support provided by FP7/ REGPOT-2012-2013.1 ( ${ }^{\circ} 316265$, BIOCAPS) and University of Vigo (PP. 00VI 131H 64102), respectively. 
Bioinspired, Biomimetic and Nanobiomaterials Volume 3 Issue BBN2
Biomineralization of marine-patterned

C-scaffolds

Rodríguez-Valencia et al.

\section{REFERENCES}

1. Costa, D. O.; Allo, B. A.; Klassen, R.; Hutter, J. L.; Dixon, S. J.; Rizkalla, A. S. Control of surface topography in biomimetic calcium phosphate coatings. Langmuir 2012, 28, 3871-3880.

2. Kokubo, T. Apatite formation on surfaces of ceramics, metals and polymers in body environment. Acta Biomaterialia 1998, 46, 2519-2527.

3. Takadama, H.; Kim, H. M.; Kokubo, T.; Nakamura, T. TEMEDX study of mechanism of bonelike apatite formation on bioactive titanium metal in simulated body fluid. Journal of Biomedical Materials Research 2001, 57, 441-448.

4. Takadama, H.; Kim, H. M.; Kokubo, T.; Nakamura, T. An $\mathrm{X}$-ray photoelectron spectroscopy study of the process of apatite formation on bioactive titanium metal. Journal of Biomedical Materials Research 2001, 55, 185-193.

5. Chen, C.; Lee, I. S.; Zhang, S. M.; Yang, H. C. Biomimetic apatite formation on calcium phosphate-coated titanium in Dulbecco's phosphate-buffered saline solution containing $\mathrm{CaCl}_{2}$ with and without fibronectin. Acta Biomaterialia 2010, 6, 2274-2281.

6. Abramovitch-Gottlib, L.; Geresh, S.; Vago, R. Biofabricated marine hydrozoans: a bioactive crystalline material promoting ossification of mesenchymal stem cells. Tissue Engineering 2006, 12, 729-739.

7. Cunningham, E.; Dunne, N.; Walker, G.; Maggs, C.; Wilcox, R.; Buchanan, F. Hydroxyapatite bone substitutes developed via replication of natural marine sponges. Journal of Materials Science: Materials in Medicine 2009, 21, 2255-2261.

8. Green, D. W. Tissue bionics: examples in biomimetic tissue engineering. Biomedical Materials 2008, 3, 1-11.

9. Green, D.; Howard, D.; Yang, X.; Kelly, M.; Oreffo, R. O. Natural marine sponge fiber skeleton: a biomimetic scaffold for human osteoprogenitor cell attachment, growth and differentiation. Tissue Engineering 2003, 9, 1159-1166.

10. López-Álvarez, M.; Pereiro, I.; Serra, J.; de Carlos, A.; González, P. Osteoblast-like cell response to macro and micro-patterned carbon scaffolds obtained from the sea rush Juncus maritimus. Biomedical Materials 2011, 6(4), 045012.

11. Huebsch, N.; Mooney, D. J. Inspiration and application in the evolution of biomaterials. Nature 2009, 462, 426-432.

12. López-Álvarez, M.; Serra, J.; de Carlos, A.; González, P. Marine-based carbon and silicon carbide scaffolds with patterned surface for tissue engineering applications. In Advances in Ceramics: Electric and Magnetic Ceramics, Bioceramics, Ceramics and Environment (Sikalidis, C. (ed.)). Rijeka: Intech, 2011, 433-451.

13. López-Álvarez, M.; de Carlos, A.; González, P.; Serra, J.; León, B. Cytocompatibility of bio-inspired silicon carbide ceramics. Journal of Biomedical Materials Research Part B Applied Biomaterials 2010, 95(1), 177-183.

14. Varela-Feria, F. M.; de Arellano-López, A. R.; MartínezFernández, J. Fabricación y propiedades del carburo de silicio biomórfico: maderas cerámicas. Boletin de la Sociedad Española de Cerámica y Vidrio 2002, 41, 377-384.

15. Wang, J. H. C.; Grood, E. S.; Florer, J.; Wenstrup, R. Alignment and proliferation of MC3T3-E1 osteoblasts in microgrooved silicone substrata subjected to cyclic stretching. Journal of Biomechanics 2000, 33, 729-735.

16. Ratner, B. D. (ed.) A history of biomaterials. In Biomaterials Science: An Introduction to Materials in Medicine (Ratner, B. D.; Hoffmann, A. S.; Schoen, F. J. (eds.)). London: Elsevier, 2004, 10-19.

17. Kuboki, Y.; Takita, H.; Kobayashi, D.; Tsuruga, E.; Inoue, M.; Murata, M.; Nagai, N.; Dohi, Y.; Ohgushi, H. BMPinduced osteogenesis on the surface of hydroxyapatite with geometrically feasible and nonfeasible structures: topology of osteogenesis. Journal of Biomedical Materials Research 1998, 39, 190-199.

18. Karageorgiou, V.; Kaplan, D. Porosity of 3D biomaterial scaffolds and osteogenesis. Biomaterials 2005, 26, 5474-5491.

19. Hayes, J. S.; Csekanska, E. M.; Richards, R. G. The cellsurface interaction. Advances in Biochemical Engineering/ Biotechnology 2012, 126, 1-31.

20. Lim, J. Y.; Donahue, H. J. Cell sensing and response to micro and nanostructured surfaces produced by chemical and topographic patterning. Tissue Engineering 2007, 13, 1879-1891.

21. Deligianni, D. D.; Katsala, N. D.; Koutsoukos, P. G.; Missirlis, Y. F. Effect of surface roughness of hydroxyapatite on human bone marrow cell adhesion, proliferation, differentiation and detachment strength. Biomaterials 2001, 22, 87-96.

22. Rosa, A. L.; Beloti, M. M.; Van Noort, R. Osteoblastic differentiation of cultured rat bone marrow cells on hydroxyapatite with different surface topography. Dental Materials 2003, 19, 768-772.

23. Smith, B. (ed.) Infrared Spectral Interpretation: A Systematic Approach. Boca Raton: CRC Press LLC, 1999.

24. Dragnea, B.; Boulmer, J.; Débarre, D.; Bourguignon, B. Growth of a $\mathrm{SiC}$ layer on $\mathrm{Si}$ (100) from absorbed propene by laser melting. Journal of Applied Physics 2001, 90, 449-455.

25. Qian, J. M.; Jin, Z. H. Preparation and characterization of porous, biomorphic $\mathrm{SiC}$ ceramic with hybrid pore structure. Journal of the European Ceramic Society 2006, 26, 1311-1316.

26. Ahmad, A. L.; Loh, M. M.; Aziz, J. A. Preparation and characterization of activated carbon from oil palm wood and its evaluation on methylene blue adsorption. Dyes and Pigments 2007, 75, 263-272.

27. Liu, Q.; Wang, S.; Zheng, Y.; Luo, Z.; Cen, K. Mechanism study of wood lignin pyrolysis by using TG-FTIR analysis. Journal of Analytical and Applied Pyrolysis 2008, 1, 170-177. 
Bioinspired, Biomimetic and Nanobiomaterials Volume 3 Issue BBN2
Biomineralization of marine-patterned

C-scaffolds

Rodríguez-Valencia et al.
28. Wang, W. L.; Ren, S. Y.; Che, Y. Z.; Chang, J. M.; Gou, J. S. Kinetics and FTIR characteristics of the pyrolysis process of poplar wood. Forestry Studies in China 2013, 15(1), 70-75.

29. Shen, W.; Li, Z.; Liu, Y. Surface chemical functional groups modification of porous carbon. Recent patents on Chemical Engineering 2008, 1(1), 27-40.

30. Otake, Y.; Jenkins, R. G. Characterization of oxygencontaining surface complexes created on a microporous carbon by air and nitric acid treatment. Carbon 1993, 31(1), 109-121.

31. Liu, H.; Yazici, H.; Ergun, C.; Webster, T. J.; Bermek, H. An in vitro evaluation of the $\mathrm{Ca} / \mathrm{P}$ ratio for the cytocompatibility of nano-to-micron particulate calcium phosphates for bone regeneration. Acta Biomaterialia 2008, 4, 1472-1479.

32. Kokubo, T.; Kim, H.; Kawashita, M. Novel bioactive materials with different mechanical properties. Biomaterials 2003, 13, 2161-2175.

33. Rodríguez-Valencia, C.; Rial, L.; Serra, J.; González, P.; Rodríguez-González, J. B.; Mateo-Mateo, C.; CorreaDuarte, M. A. Tuning the biomineralization process for controlling the nucleation and oriented growth of $\mathrm{Ca}-\mathrm{P}$ crystals onto functionalized carbon nanotubes. Journal of Physical Chemistry C 2012, 116(5), 3400-3404.

\section{WHAT DO YOU THINK?}

To discuss this paper, please email up to 500 words to the managing editor at bbn@icepublishing.com

Your contribution will be forwarded to the author(s) for a reply and, if considered appropriate by the editor-inchief, will be published as a discussion in a future issue of the journal.

ICE Science journals rely entirely on contributions sent in by professionals, academics and students coming from the field of materials science and engineering. Articles should be within 5000-7000 words long (short communications and opinion articles should be within 2000 words long), with adequate illustrations and references. To access our author guidelines and how to submit your paper, please refer to the journal website at www.icevirtuallibrary.com/bbn 